Ann. Zootech., I975, 24 (3), 553-558.

NOTE

\title{
NOMBRE DE TÉTÉES ET TEMPS DE PÂTURAGE DES VEAUX DANS LES TROUPEAUX DE VACHES ALLAITANTES
}

\author{
P. LE NEINDRE et M. PETIT \\ avec la collaboration technique de H. Dubroeuco \\ Laboratoire de la Production de Viande, \\ Centre de Recherches de Clermont-Ferrand, I. N. R. A., \\ Theix, Saint Genès Champanclle, \\ 63110 Beatmont
}

RÉSUMÉ:

Nous avons observé l'emploi du temps sur 24 heures pendant 7 journées de 10 à 15 vaches allaitantes avec leur veau.

La répartition des tétées durant le nycthémère varie avec l'âge du veau. Les tétées nocturnes importantes dans le jeune âge diminuent par la suite pour réapparaître à l'automne associées à un pâturage nocturne. Le nombre de tétées journalières apparaît lié à l'âge du veau et indépendant de la production laitière. Le temps de pâturage des veaux dépend à la fois de l'âge du veau et de la production laitière de la mère; le veau serait donc capable de compenser une consommation de lait faible par une augmentation de la consommation d'herbe.

\section{INTRODUCTION}

La fréquence des tétées des veaux en liberté avec leurs mères a fait l'objet d'un certain nombre d'observations effectuées dans la plupart des cas sur des races anglo-saxonnes (DrEWRY et al., I959; Hutchison et al., I962 ; Walker, I962 ; Hafez et Lineweaver, i968 ; Ewbank, 1969 ; Rugh et WILSON, 1971). En revanche, le temps de pâturage des veaux a été peu observé (HuTCHison et al., 1962, sur zébu; Petrt, 1972, sur vache $A u b r a c$ ). Il n'existe, à notre connaissance aucune étude simultanée du nombre de tétées et du temps de pâturage des veaux cn rclation avec leur âge et la quantité de lait dont ils disposent.

Une première série d'observations (P. LE NEINDRE, 1973) effectuées sur I 7 heures, de l'aube au crépuscule, nous avait permis d'étudier l'évolution avec l'âge de la fréquence des tétées. 
Dans la présente étude, de nouvelles observations nous permettront de décrire l'emploi du temps complet des veaux sur 24 heures et de préciser, selon le stade de lactation, l'influence de la production laitière des vaches sur le nombre de tétées et sur le temps de pâturage de leurs veaux.

\section{MA'TÉRIEL E'T MÉTHODES}

Pendant 4 journées, (27 février, 9 mars, 7 avril, 27 mai), nous avons observé l'emploi du temps de 5 vaches Charolaises (date moyenne de vêlage: I 7 février), de 5 vaches Salers (I I mars) et de leurs veaux, hivernant en stabulation libre et recevant deux repas de foin par jour; puis pendant trois journées ( 7 juillet, 6 septembre, 27 septembre), les ro vaches précédentes plus 5 vaches A ubrac (date moyenne de vêlage: 3 I mars), cn liberté avec leurs veaux au pâturage. Tous les veaux étaient issus de taureaux Charolais.

Les observations étaient continues, débutaient au lever du jour et se terminaient à l'aube du lendemain ; du crépuscule à l'aube, une torche électrique était utilisée pendant de courts instants. L'activité de pâturage des animaux était notée individuellement à intervalle de to minutes. Les définitions utilisées et le calcul des temps de pâturage journaliers sont identiques à ceux de M. Petit ( I 969). Les heures de début et de fin des tétées ont été notées systématiquement : on a considéré qu'un veau tétait quand il avait la tête sous la mamelle. Pour calculer le nombre de tétées, nous n'avons pas compté celles qui duraient moins de 2 minutes et nous avons considéré comme une seule tétée deux tétées consécutives séparées par moins de ${ }_{5}$ minutes.

En plus de ces observations, la production laitière des vaches a été mesurée régulièrement toutes les 3 semaines par pesées des veaux avant et après la tétéc, deux fois en 24 hcures.

\section{RÉSULTATS}

\section{Les tétées}

Comme Ewbank (i969), Wagnon (1963), Hafez et Linweaver (I968), Hutchinson et al. (I962), nous observons des tétées à toute heure surtout chez les tout jeunes veaux en stabulation libre (fig. I). Cependant, au lever du soleil, se situe toujours une période intense de tétées ; 80 à 100 p. Ioo des veaux ont tété durant les 3 heures encadrant le lever du soleil. Au pâturage, pour des veaux plus âgés, les cycles de tétées coïncident avec les cycles de pâturage du troupeau. A l'automne, comme M. Petit (1972), nous observons des tétées nocturnes associées à une période de pâturage (fig. I).

TABLEAU I

Données moyennes

\begin{tabular}{|c|c|c|}
\hline & Veaux jeunes & Veaux âgés \\
\hline Mode de conduite & Stabulation libre & Pâturage \\
\hline Nombre d'observations & 33 & 45 \\
\hline Age des veaux (jours) $\ldots . . \ldots \ldots$ & $39,5 \pm 24,1$ & $166,7 \pm 1,1,4$ \\
\hline Quantité de lait bu $(\mathrm{kg}) \ldots \ldots \ldots$ & $7,1 \pm 3,0$ & $7,2 \pm 2, \underline{2}$ \\
\hline Nombre de tétées ............. & $6,0 \pm 3,0$ & $2,9 \pm 1,0$ \\
\hline Temps de tétée journalier (mn) & $62,6 \pm 18,1$ & $35,8 \pm 12,1$ \\
\hline Temps par tétée $(\mathrm{mn}) \quad \ldots \ldots$ & $11,0 \pm 3,1$ & $12,0 \pm 2,3$ \\
\hline Temps de pâturage $(h) \quad \ldots$ & & $6,35 \pm 1,75$ \\
\hline
\end{tabular}




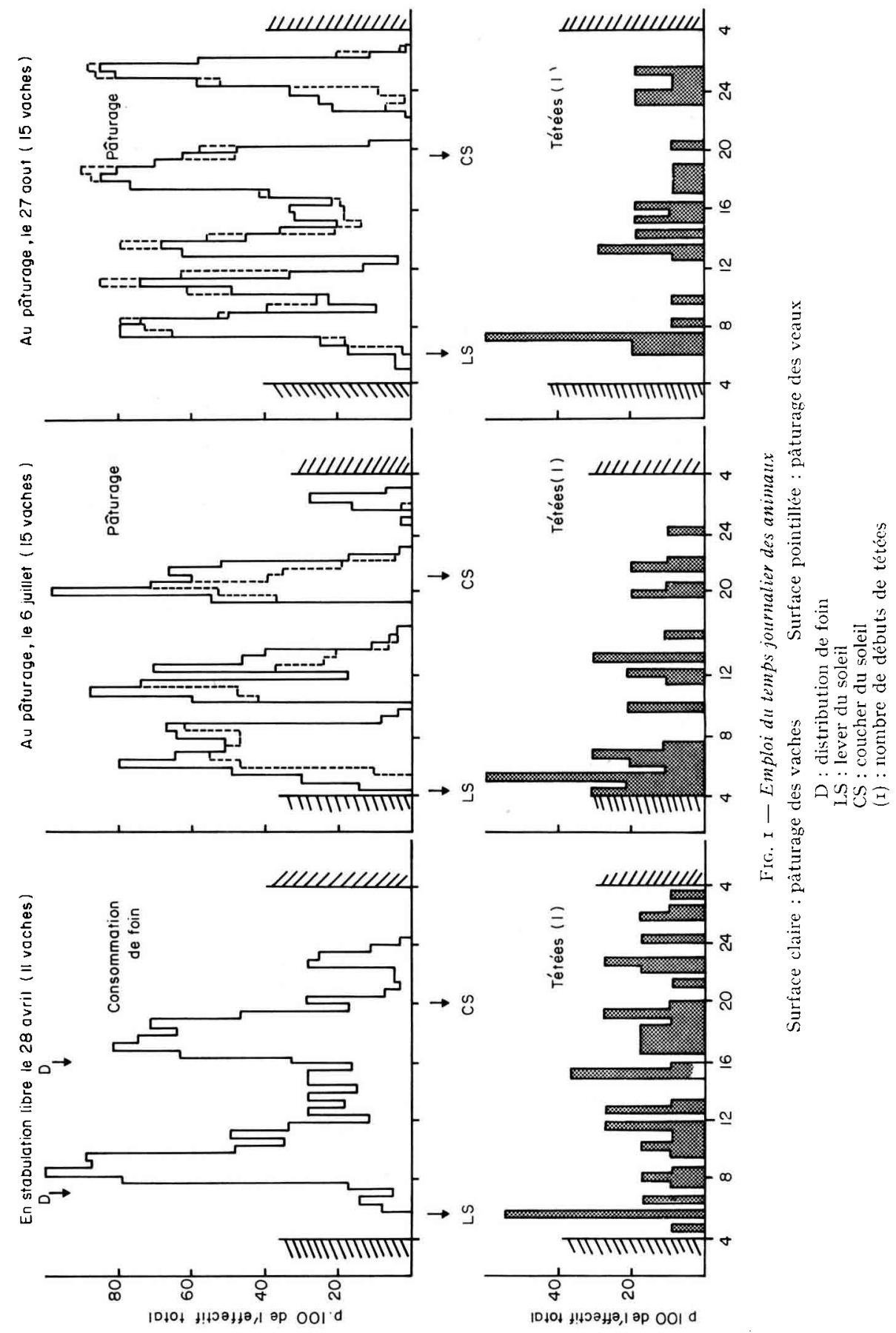


P. IE NEINDRE, M. PETIT

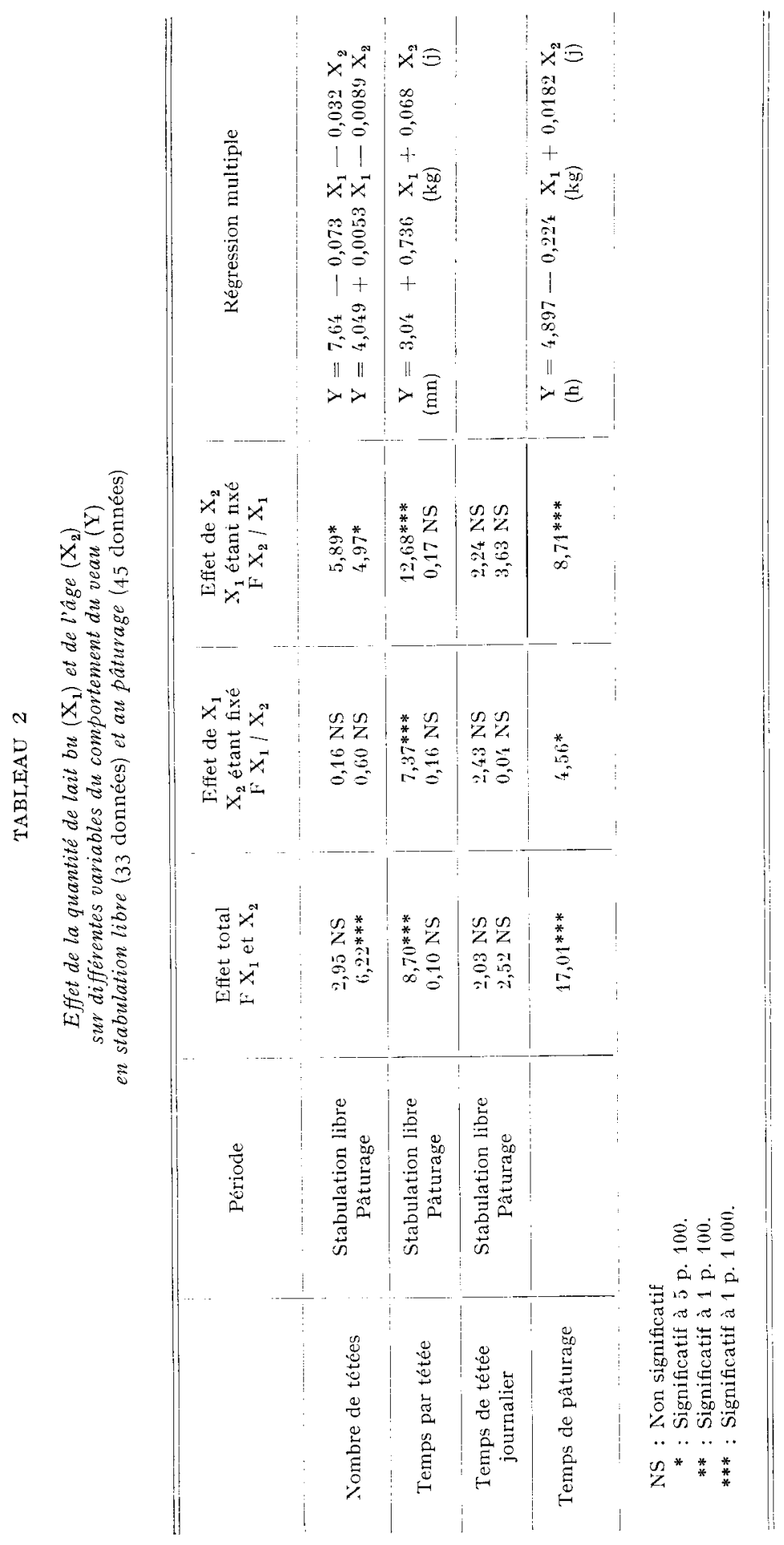


Globalement, le rapport des tétées nocturnes sur le total des tétées varie de $\mathbf{~} 6,3$ à 50 p. Ioo entre les heures légales de coucher ct lever du soleil ; il est d'autant plus important que les nuits sont longues. Si on ne tient compte que de la période d'obscurité complète, ce rapport ne varie plus que de $4, \mathrm{I}$ à 29,4 p. 1 oo.

Le nombre de tétées par 24 heures diminue lorsque le veau avance en âge (tabl. I) et de façon beaucoup plus importante pendant les deux premiers mois que par la suite (fig. 2). Au même âge, les veaux tètent le même nombre de fois quelle que soit la production laitière des mères (tabl. 2.)

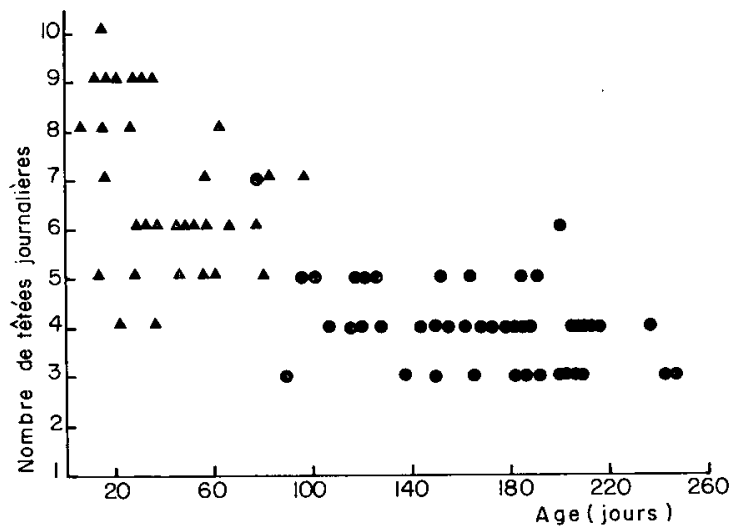

FIG. 2. - Evolution du nombre des tétées journalières avec l'âge

(Chaque point représente le résultat d'un jour d'observation d'un veau)

- en stabulation libre

- au pâturage

Melton et al. (1967) supposent que la production laitière des vaches allaitant des mâles est supérieure à celle allaitant des femelles parce que les veaux mâles tètent plus souvent. En fait, comme RugH et WiLson (I97I), nous n'observons pas de différences dans le nombre des tétées journalières pour des mâles et des femelles de même âge.

Pour les jeunes veaux, en stabulation libre, la durée moyenne d'une tétée augmente avec l'âge du veau et la production laitière (tabl. 2). A l'herbe avec des veaux plus âgés, elle ne dépend ni de l'âge ni de la production laitière. Le temps total de tétée journalier cépend peu de l'âge et de la production laitière (tabl. 2).

\section{Le temps de pâturage}

Les veaux pâturent en général en même temps que les vaches. De même que M. PeTit (I972), nous observons un cycle de pâturage nocturne important quand les veaux sont âgés (fig. I).

Comme Hutchison et al. (1962), M. Petit (1972), nous trouvons que plus un veau cst âgé, plus il pâture longtemps, mais également que, au même âge, comme l'a déjà rcmarqué M. Petir (1972), une consommation de lait faible entraîne un temps de pâturage élevé (tabl. 2).

\section{CONCLUSION}

Le temps de pâturage journalier du veau est fonction à la fois de son âge et de la production laitière de la mère. Au même âge, le veau peut compenser en partie une consommation en lait faible par un temps de pâturage plus élevé et donc une consommation d'herbe plus importante. 
Le nombre de tétées journalier diminue avec l'âge du veau, alors que la rlurée de chaque tétée augmente lorsque le veau est jeune puis reste constante.

L'emploi du temps journalier des veaux est semblable à celui observé par MI. PETIT (I972). 11 apparaît que les activités nocturnes varient avec la durée de la nuit et l'âge du veau, ce qui rend difficile l'interprétation de l'évolution des paramètres du comportement à partir d'observations effectuées uniquement lans la période diurne.

$$
\text { Reçu pour publication en mars } 1975 .
$$

\section{SUMMARY}

\section{NUMBER OF SUCKLINGS \\ AND GRAZING TIME OF CAIVES IN NURSING COW HERDS}

The grazing and suckling behaviour of nursing cows and their calves was recorded. The observations were made on ten cows housed during winter and fifteen cows on pasture during summer. Observation periods were : 4 periods of 24 hours in winter and 3 periods of 24 hours in summer.

The distribution of suckling periods over 24 hours varied according to the age of the calves (fig. I). The young calves often suckled during the night, but this nocturnal suckling stopped when the animals became older. However, during autumn when the dams started grazing during the night, the nocturnal sucklings of the calves began again.

The number of sucklings over a period of 24 hours decreased with increasing age of the calves (fig. 2, table I) and was independent of the dam's milk production. The total grazing time of the calves both depended on their age and their milk intake. They were thus able to compensate for a low milk supply by increasing their grass intake.

\section{RÉFÉRENCES BIBLIOGRAPHIQUES}

DREWRY K. J., Brown C. H., HoneA R. S., 1959. Relationship among factors associated with mothering ability in beef cattle. J. Anim. Sci., 18, $938-946$.

EWBANK R., 1969. The frequency and duration of the nursing periods in single-suckled Hereford beef cows. Brit. Vet. J., 125, IX-X.

Hafez I..S. E., Lineweaver J. A., 1968. Suckling Behaviour in Natural and Artificially Fed Neonate Calves. Zeitsch. fur Tierpsych., 25, 187-r98.

Hutchison H. C., Woof R., Mabon R. M., Salehe I., Robb J. M., ig62. A study of the habits of Zebu cattle in Tanganyika. J. Agric. Sci., 59, 301-3I7.

Le Neindre P., I973. Observations sur l'estimation de la production laitière des vaches allaitantes. Ann. Zootech., 22, 4r3-422.

Melton R. A., Riggs J. K., Nelson L. A., Cartwrigit T. C., i967. Milk production and calf gains of Angus Charolais and Hereford cows. J. Anim. Sci, 26, 804-809.

Petit M., Ig69. Méthode d'observation de l'emploi du temps des troupeaıx de vaches allaitantes au pâturage. Ann. Zootech., 18, 221-226.

Petit M., 1972. Emploi du temps des troupeaux de vaches mères et de leurs veaux sur les pâturages d'altitude de l'Aubrac. Ann. Zootech., 21, 5-27.

Rugh M. C., Wilson L. L., I97I. Influence of energy intake, cow body size and calf sex on certain behavioral traits of Angus-Holstein cows and their progeny. J. Anim. Sci., 32, 728-73I.

Wagnon K. A. 1963. Behaviour of beef cows on a california range. Calufornia Agr. Exp. Stat. Bull., $799,58 \mathrm{p}$.

WALKER D, E., 1962. Suckling and grazing behaviour of beef heifers and calves. N. Z. J. Agric. Res., 5, 331-338. 